\title{
The miracle cure
}

This Editor's Choice article published online on 19 September (BMJ 2019;366:15605, doi:10.1136/bmj.15605) and in print on 21 September referred to the Academy of Medical Sciences instead of the Academy of Medical Royal Colleges. The online version has now been corrected. 